\title{
On First Hermitian-Zagreb Matrix and Hermitian-Zagreb Energy
}

\author{
A. Bharali \\ Department of Mathematics, Dibrugarh University, Assam, India \\ *Corresponding Author: a.bharali@dibru.ac.in, Tel.: +91-9954293291
}

Available online at: www.isroset.org

Received: 17/May/2018, Revised: 28/May/2018/2018, Accepted: 16/Jun/2018, Online: 30/Jun/2018

\begin{abstract}
A mixed graph is a graph with edges and arcs, which can be considered as a combination of an undirected graph and a directed graph. In this paper we propose a Hermitian matrix for mixed graphs which is a modified version of the classical adjacency matrix related to the first Zagreb index. We also establish some results related to this newly proposed matrix. Finally we present some results on the Hermitian-Zagreb energy.
\end{abstract}

Keywords - first Zagreb index, Hermitian adjacency matrix, energy.

MSC: 05C50; 05C07; 05C31

\section{INTRODUCTION}

In chemical graph theory, topological index or molecular descriptor is a numerical value associated with chemical constitution for correlation of chemical compounds with various physio-chemical properties (for example: boiling point, enthalpy of vaporization, stability and so on) or biological activities. Over the period of last few decades, many topological indices were defined and studied based on degree, distance between the vertices etc. An account of some degree-based topological indices may be found in [8]. In a paper published in 1972, Trinajstić and Gutman [7] discussed the dependency of total electron $\pi$-energy of alternant hydrocarbons on molecular structure in the form of a graph $G$ and found two terms that occur in its approximate expressions which are of the form:

$$
\begin{aligned}
& M_{1}(G)=\sum_{u v \in E(G)}\left(d_{u}+d_{v}\right) \\
& M_{2}(G)=\sum_{u v \in E(G)} d_{u} d_{v},
\end{aligned}
$$

where $d_{u}$ is the degree of the vertex $u$ in $G$. in case of mixed graph $M$ it is the degree of $u$ in the underlying graph of $M$. Immediately, they were proposed to measure the branching of carbon-atom skeleton [6] for their proportional behaviour with the extent of branching. Few years later only, Balaban et al., [1] used the terminology first and second Zagreb indices' (more precisely Zagreb group indices) for $\mathbf{M}_{1}$ and $\mathrm{M}_{2}$ respectively and called them as topological indices.

In 2005, Rodríguez initiated the study of spectral properties of topological indices by associating weighted adjacency matrices to the indices [13]. Since then a reasonable number of works were reported on the study of spectral properties of the topological indices [2], [3], [14], [15]. Recently in 2018, Rad et al., communicated some results on Zagreb energy and Zagreb Estrada index of graphs [12]. In these studies researchers consider only undirected graphs, but in last few years some attempts are also reported for oriented as well as mixed graphs for Randić index [4], [11].

The Hermitian-adjacency matrix of a mixed graph $M$ of order $n$ is the $n \times n$ matrix $H(M)=\left(h_{k l}\right)$, where $h_{k l}=$ $h_{l k}=\boldsymbol{i}(\boldsymbol{i}=\sqrt{ }-1)$ if $\left(\mathrm{v}_{\mathrm{k}}, \mathrm{v}_{\mathrm{l}}\right)$ is an $\operatorname{arc}$ of $M, h_{k l}=h_{l k}=1$ if $v_{k} v_{l}$ is an undirected edge of $M$, and $h_{k l}=0$ otherwise. Obviously, $H(M)=H(M)^{*}:=\overline{H(M)}^{T}$. Thus all its eigenvalues are real. This matrix was introduced by Liu and $L i$ in [10] and independently by Guo and Mohar in [5]. From the above we can see that if we add a first Zagreb weight to every edge and $\operatorname{arc}$ in $M$, then we can get a new weighted Hermitianadjacency matrix. What are the properties of this new matrix? This motivates us to define the first HermitianZagreb matrix, $H_{Z}(M)$ of a mixed graph $M$. Let us denote and $\operatorname{arc} v_{p} \rightarrow v_{q}$ by $(p, q)$ and an edge (undirected or both directed) $v_{p}-v_{q}$ or $v_{p} \leftrightarrow v_{q}$ by simply $p q$. The matrix $H_{Z}(M)$ can be defined as follows:

$$
H_{Z}(M)=\left(h_{z}\right)_{p q}=\left\{\begin{array}{cc}
i\left(d_{p}+d_{q}\right) & \text { if }(p, q) \in E(M) \\
-i\left(d_{p}+d_{q}\right) & \text { if }(q, p) \in E(M) \\
d_{p}+d_{q} & \text { if } p q \in E(M) \\
0 & \text { Otherwise. }
\end{array}\right.
$$

In 1978, Gutman introduced the concept of graph energy [9]. Since then many extensions of this graph invariant have been proposed. In this paper we also define an extension of this graph invariant based on the eigenvalues of the above proposed first Hermitian-Zagreb matrix.

The rest of the paper is organized as follows. In the next section we study the spectral properties of the first Hermitian-Zagreb matrix. In section 3, we report some 
results on the first Hermitian-Zagreb energy. In section 4, conclusions are made.

\section{PROPERTIES OF THE FIRST HERMITIAN- ZAGREB MATRIX}

In this section first we analyze the matrix $H_{Z}(M)$ in terms of its structure and spectral properties. It is clear that $H_{Z}(M)$ is a hermitian matrix and if the graph $M$ is undirected then $H_{Z}(M)=Z(M)$, where $Z(M)$ is the symmetric matrix proposed by Rad et al., in [12]. Again if $M$ is an oriented graph $H_{Z}(M)=i H_{s}(M)$, where $H_{S}(M)$ is a skew-symmetric matrix.

Theorem 2.1: Let $H_{Z}(M)$ be the first Hermitian-Zagreb matrix and $H Z(G)$ be the hyper Zagreb index of a graph $G$. Then

a) $\operatorname{Trace}\left(H_{Z}(M)=0\right.$.

b) If $M_{U}$ be the underlying graph of $M$ and $M_{U}$ is rregular, then $H_{Z}(M)=2 r H(M)$.

c) If $\lambda_{1}, \lambda_{2}, \lambda_{3}, \ldots, \lambda_{\mathrm{n}}$ be the eigenvalues of $H_{Z}(M)$, then

i. $\quad$ they are all real, also $\sum_{p=1}^{n} \lambda_{p}=0$.

ii. $\quad \sum_{p=1}^{n} \lambda_{p}^{2}=2 H Z\left(M_{U}\right)$ and $\sum_{p \neq q}^{n} \lambda_{p} \lambda_{q}=$ $2 H Z\left(M_{U}\right)$

Proof: Expression (a) and (b) can easily be proved from the definition of the matrix $H_{Z}(M)$. To prove the first result of (c) we can use the fact that $H_{Z}(M)$ is Hermitian, and the eigenvalues of a Hermitian matrix are always real. And also $\sum_{p=1}^{n} \lambda_{p}=\operatorname{Trace}\left(H_{Z}(M)\right)$.

Again to prove the second result of (c), we use the fact that $\sum_{p=1}^{n} \lambda_{p}{ }^{2}=\operatorname{Trace}\left(H_{z}(M)^{2}\right)$. So, we have

$$
\begin{aligned}
\sum_{p=1}^{n} \lambda_{p}{ }^{2}= & \sum_{k=1}^{n} \sum_{l=1}^{n}\left(h_{z}\right)_{k l}\left(h_{z}\right)_{l k}{ }^{*} \\
= & 2 \sum_{(l, k) \epsilon E(M)}\left(h_{z}\right)_{k l} \overline{\left(h_{z}\right)_{k l}}+ \\
& 2 \sum_{l k \in E(M)}\left(h_{z}\right)_{k l} \overline{\left(h_{z}\right)_{k l}}
\end{aligned}
$$

In the above expression we have considered only those values of $l$ and $k$ for which either $(l, k)$ or $(k, l)$ is an arc, or $l k$ is an edge. This is because, otherwise $\left(h_{z}\right)_{k l}=0$, and it is not going to contribute anything to the summation. If $(l, k)($ or $(k, l))$ is an arc then $\left(h_{z}\right)_{k l}=i\left(d_{l}+d_{k}\right)$ (or $\left.-i\left(d_{l}+d_{k}\right)\right)$. Again if $l k$ is an edge, then $\left(h_{z}\right)_{k l}=\left(d_{l}+\right.$ $\left.d_{k}\right)$. In all the cases it is easy to verify that $\left(h_{z}\right)_{k l} \overline{\left(h_{z}\right)_{k l}}=$ $\left(d_{l}+d_{k}\right)^{2}$. And hence $\sum_{p=1}^{n} \lambda_{p}{ }^{2}=2 \sum_{l k \in E\left(M_{U}\right)}\left(d_{l}+\right.$ $\left.d_{k}\right)^{2}=2 H Z\left(M_{U}\right)$. The last part (c) can be easily proved by using the identity $\left(\sum_{p=1}^{n} \lambda_{p}\right)^{2}=\sum_{p=1}^{n} \lambda_{p}{ }^{2}+\sum_{p \neq q}^{n} \lambda_{p} \lambda_{q}$.

Theorem 2.2: Let $\lambda_{1}, \lambda_{2}, \lambda_{3}, \ldots, \lambda_{\mathrm{n}}$ be the eigenvalues of $H_{Z}(M)$ and $\lambda_{\min }=\min _{1 \leq p \leq n} \lambda_{p}$. Then

$$
\lambda_{\min }{ }^{2} \geq \frac{2 H Z\left(M_{U}\right)}{n(n-1)}
$$

where $H Z\left(M_{U}\right)$ is the Hyper-Zagreb index of the underlying graph $M_{U}$ of mixed graph $M$.

Proof: We start with the identity that

$\sum_{p=1}^{n}\left(\lambda_{p}-\lambda_{\text {min }}\right)=\sum_{p=1}^{n} \lambda_{p}-n \lambda_{\text {min }}=-n \lambda_{\text {min }}$, as from Theorem 2.1(a) $\sum_{p=1}^{n} \lambda_{p}=0$. Squaring both sides we have,

$$
\sum_{p=1}^{n}\left(\lambda_{p}-\lambda_{\text {min }}\right)^{2}+\zeta=\left(n \lambda_{\text {min }}\right)^{2},
$$

where $\zeta=\sum_{p \neq q}^{n}\left(\lambda_{p}-\lambda_{\text {min }}\right)\left(\lambda_{q}-\lambda_{\text {min }}\right)$ is a non-negative number. Hence

$$
\begin{aligned}
& \sum_{p=1}^{n}\left(\lambda_{p}-\lambda_{\text {min }}\right)^{2} \leq n^{2} \lambda_{\text {min }}{ }^{2} \\
= & \sum_{p=1}^{n} \lambda_{p}{ }^{2}+n \lambda_{\text {min }}{ }^{2} \leq n^{2} \lambda_{\text {min }}{ }^{2} .
\end{aligned}
$$

Hence the result follows from Theorem 2(c).

Theorem 2.3: Let $\lambda_{1}, \lambda_{2}, \lambda_{3}, \ldots, \lambda_{\mathrm{n}}$ be the eigenvalues of $H_{Z}(M)$ and $\lambda_{\max }=\max _{1 \leq p \leq n} \lambda_{p}$. Then

$$
\lambda_{\max } \leq \sqrt{\frac{2(n-1) H Z\left(M_{U}\right)}{n}}
$$

where $H Z\left(M_{U}\right)$ is the Hyper-Zagreb index of the underlying graph $M_{U}$ of mixed graph $M$. Equality holds iff $H Z\left(M_{U}\right)$ has at most two distinct eigenvalues.

Proof: We know that $\sum_{p=1}^{n} \lambda_{p}=0$ and $\sum_{p=1}^{n} \lambda_{p}{ }^{2}=$ $2 H Z\left(M_{U}\right)$. Let $\lambda_{\max }=\lambda_{q}$. Applying Cauchy-Schwarz inequality on the sets $\left\{\lambda_{1}, \lambda_{2}, \ldots, \lambda_{q-1}, \lambda_{q+1}, \ldots, \lambda_{n}\right\}$ and $\{1,1,1, \ldots, 1\}$, each of these sets contains $(n-1)$ elements. We have

$$
\begin{aligned}
& \left(\sum_{p=1, p \neq q}^{n}\left|\lambda_{p}\right| \cdot 1\right)^{2} \leq(n-1) \sum_{p=1, p \neq q}^{n}\left|\lambda_{p}\right|^{2} \\
& \Rightarrow \lambda_{\max }{ }^{2} \leq(n-1)\left(2 H Z\left(M_{U}\right)-\lambda_{\max }{ }^{2}\right) \text {, }
\end{aligned}
$$

On simplification we have the result.

Now we will give an expression of determinant and the characteristic polynomial of $H_{Z}(M)$. To obtain the expression we first need to introduce some basic definitions. The value of a mixed walk $W=v_{1} v_{2} \ldots v_{k}$ is $W=h_{z}(W)=$ $\left(h_{z}\right)_{12}\left(h_{z}\right)_{23}\left(h_{z}\right)_{34} \ldots\left(h_{z}\right)_{(k-1) k}$. A mixed walk is called positive (negative) if this value for that walk is positive (negative). For sign we will consider the sign of the value ignoring real or complex. Note that for one direction the value of a mixed walk or a mixed cycle is $\alpha$, then for the reversed direction its value is $\bar{\alpha}$. If each mixed cycle is positive (resp. negative) in a mixed graph $M$, then $M$ is positive (resp. negative). A mixed graph $M$ is called an elementary graph if every component of $M$ is an edge, an arc or a mixed cycle, where every edge-component in $M$ is defined to be positive. A real spanning elementary subgraph of a mixed graph $M$ is an elementary subgraph such that it contains all vertices of $M$ and all its mixed cycles are real.

Now we will give two results which are similar to those in [11]. The proofs of these results are avoided because they are similar to the ones done by $L u$ et al., in [11].

Theorem 2.4: Let $H_{Z}(M)$ be the first Hermitian-Zagreb matrix of a mixed graph $M$ of order $n$. Then

$$
\operatorname{det}\left(H_{Z}(M)\right)=\sum_{M^{\prime}}(-1)^{r\left(M^{\prime}\right)+l\left(M^{\prime}\right)} 2^{s\left(M^{\prime}\right)} W\left(M^{\prime}\right),
$$

where the summation is over all real spanning elementary subgraphs $M^{\prime}$ of $M, \mathrm{r}\left(M^{\prime}\right)=\mathrm{n}-\mathrm{c}\left(M^{\prime}\right), \mathrm{c}\left(M^{\prime}\right)$ denotes the number of components of $M^{\prime}, l\left(M^{\prime}\right)$ denotes the number of negative mixed cycles of $M^{\prime}, \mathrm{s}\left(M^{\prime}\right)$ denotes the number of 
mixed cycles with length $\geq 3$ in $M^{\prime}$, $W\left(M^{\prime}\right)=\prod_{(l, k) \text { or } l k \in E\left(M^{\prime}\right)}\left(d_{l}+d_{k}\right)$.

Theorem 2.5: For a mixed graph $M$, if we consider the $\mathrm{k}^{\text {th }}$ coefficients of $H_{Z}$-characteristic polynomial to be $a_{k}$, then

$$
(-1)^{k} a_{k}=\sum_{M^{\prime}}(-1)^{r\left(M^{\prime}\right)+l\left(M^{\prime}\right)} 2^{s\left(M^{\prime}\right)} W\left(M^{\prime}\right),
$$

where he summation is over all real elementary subgraphs $M^{\prime}$ with order $k$ of $M, \mathrm{r}\left(M^{\prime}\right)=\mathrm{k}-\mathrm{c}\left(M^{\prime}\right), \mathrm{c}\left(M^{\prime}\right)$ denotes the number of components of $M^{\prime}, l\left(M^{\prime}\right)$ denotes the number of negative mixed cycles of $M^{\prime}, \mathrm{s}\left(M^{\prime}\right)$ denotes the number of mixed cycles with length $\geq 3$ in $M^{\prime}$, $W\left(M^{\prime}\right)=\prod_{(l, k) \text { or } l k \epsilon E\left(M^{\prime}\right)}\left(d_{l}+d_{k}\right)$.

Theorem 2.6: If $M$ is a positive mixed graph, then $H_{Z}(M)$ spectrum of $M$ and $M_{U}$ are same.

Proof: It can be proved from the fact the for a positive graph $M$,

$(-1)^{r\left(M^{\prime}\right)+l\left(M^{\prime}\right)} 2^{s\left(M^{\prime}\right)} W\left(M^{\prime}\right)=(-1)^{r\left(M^{\prime}\right)} 2^{s\left(M^{\prime}\right)} W\left(M^{\prime}\right)=$ $(-1)^{r\left(M_{U}^{\prime}\right)} 2^{s\left(M_{U}^{\prime}\right)} W\left(M_{U}^{\prime}\right)$.

\section{FIRST HERMITIAN-ZAGREB ENERGY}

Analogous to the graph energy proposed by Gutman, we define the first Hermitian-Zagreb energy, $E H_{z}(M)$ of a mixed graph $M$ as follows. If $\lambda_{1}, \lambda_{2}, \lambda_{3}, \ldots, \lambda_{\mathrm{n}}$ be the eigenvalues of $H_{Z}(M)$, then $E H_{z}(M)=\sum_{p=1}^{n}\left|\lambda_{p}\right|$.

Lemma 3.1: Let $M$ be a mixed graph of order $n$ and $\lambda_{1}, \lambda_{2}$, $\lambda_{3}, \ldots, \lambda_{\mathrm{n}}$ be the Hermitian-Zagreb spectrum of $H_{Z}(M)$. Then $\left|\lambda_{1}\right|=\left|\lambda_{2}\right|=\cdots=\left|\lambda_{n}\right|$ iff there exists some constant $\eta$, such that $\left|\lambda_{p}\right|^{2}=\eta$ for all $p$ and $H_{Z}(M)^{2}=\eta I_{n}$.

Proof: Given that $\lambda_{1}, \lambda_{2}, \lambda_{3}, \ldots, \lambda_{\mathrm{n}}$ is the Hermitian-Zagreb spectrum of $H_{Z}(M)$. Then there exists a unitary matrix $U$ such that

$$
\mathrm{U}^{*} H_{Z}(M) \mathrm{U}=\mathrm{UH}_{Z}(M)^{*} \mathrm{U}^{*}=\operatorname{diag}\left(\lambda_{1}, \lambda_{2}, \lambda_{3}, \ldots, \lambda_{n}\right) \text {. }
$$

Now if $\left|\lambda_{1}\right|=\left|\lambda_{2}\right|=\cdots=\left|\lambda_{n}\right|$ then clearly there exists some constant $\eta$, such that $\left|\lambda_{p}\right|^{2}=\eta$ for all $p$. Also $\left(\mathrm{U}^{*} H_{Z}(M) \mathrm{U}\right)\left(\mathrm{U}^{*} H_{Z}(M)^{*} \mathrm{U}\right)=\operatorname{diag}\left(\left|\lambda_{1}\right|^{2},\left|\lambda_{2}\right|^{2}, \ldots\right.$, $\left.\left|\lambda_{n}\right|^{2}\right)$. As $H_{Z}(M)$ is Hermitian, we can write the above expression as $\mathrm{U}^{*} H_{Z}(M)^{2} \mathrm{U}=\eta I_{n}$. Then multiplying both sides of this expression by $\mathrm{U}$ and $\mathrm{U}^{*}$, we have $H_{Z}(M)^{2}=$ $\eta I_{n}$, where $\eta=\left|\lambda_{p}\right|^{2}$ for all $p$.

Now we establish some sharp bounds of $E H_{z}(M)$ and using Lemma 3.1 we can obtain the equality conditions for these bounds.

Theorem 3.2: Let $M$ be a mixed graph of order $n$, and $\lambda_{1}, \lambda_{2}$, $\lambda_{3}, \ldots, \lambda_{\mathrm{n}}$ be the Hermitian-Zagreb spectrum of $H_{Z}(M)$. If $\rho=\left|H_{Z}(M)\right|$ then

$\sqrt{2 H Z\left(M_{U}\right)+n(n-1) \rho^{2 / n}} \leq E H_{Z}(M) \leq \sqrt{2 n H Z\left(M_{U}\right)}$, equalities hold iff there exists some constant $\eta$, such that $\left|\lambda_{p}\right|^{2}=\eta$ for all $p$ and $H_{Z}(M)^{2}=\eta I_{n}$.

Proof: From Theorem 2.1(c), we know that $\sum_{p=1}^{n}\left|\lambda_{p}\right|^{2}=$ $2 H Z\left(M_{U}\right)$. Now using Cauchy-Schwarz inequality we have

$$
\left(\sum_{p=1}^{n}\left|\lambda_{p}\right| .1\right) \leq \sqrt{n \sum_{p=1}^{n}\left|\lambda_{p}\right|^{2}}=\sqrt{2 n H Z\left(M_{U}\right)} .
$$

On the other hand $\left|E H_{z}(M)\right|^{2}=\left(\sum_{p=1}^{n}\left|\lambda_{p}\right|\right)^{2}=$ $\sum_{p=1}^{n}\left|\lambda_{p}\right|^{2}+\sum_{p \neq q}^{n}\left|\lambda_{p} \lambda_{q}\right|$

Using AM-GM inequality, we can show that $\sum_{p \neq q}^{n}\left|\lambda_{p} \lambda_{q}\right| \geq \mathrm{n}(\mathrm{n}-1) \rho^{2 / n}$

From expression (3.1) and (3.2) we have $\sqrt{2 H Z\left(M_{U}\right)+n(n-1) \rho^{2 / n}} \leq E H_{z}(M)$.

Now for equality in both the bounds we must have equality in the corresponding Cauchy-Schwarz inequality and AM-GM inequality. And in the both inequalities, equality holds iff $\left|\lambda_{1}\right|=\left|\lambda_{2}\right|=\cdots=\left|\lambda_{n}\right|$. Now using Lemma 3.1 we have the desired result.

\section{CONCLUSIONS}

In this paper it is tried to extend the classical adjacency matrix for mixed graphs, corresponding to first Zagreb index and then we study this matrix for its spectral properties. An extension of the graph invariant "graph energy" is also proposed. We call it "first Hermitian-Zagreb energy". We establish few bounds of this novel extension of graph energy. In future work we will try to study similar matrix for the second Zagreb index. We also plan to study this matrix for oriented graphs for which we commonly define a skew symmetric matrix.

\section{Acknowledgement}

The author is grateful to all the anonymous reviewers for their valuable suggestions to improve the manuscript.

\section{REFERENCES}

[1] A. T. Balaban, et al., "Topological indices for structure-activity correlations", Topics Curr. Chem., Vol. 114, pp. 21-55, 1983.

[2] X. Chen, "On ABC eigenvalues and ABC energy", Lin. Alg. Appl., Vol. 544, pp. 141-157, 2018.

[3] E. Estrada, "The ABC Matrix", J Math Chem, Vol. 55, pp. 10211033, 2017.

[4] R. Gu, et al., "Skew Randić matrix and Skew Randić energy", Trans. Combin., Vol. 5, No. 1, pp. 1-14, 2016.

[5] K. Guo, B. Mohar, "Hermitian Adjacency Matrix of Digraphs and Mixed Graphs", Journal of Graph Theory, Vol. 85, No. 1, pp. 217-248, 2017.

[6] I. Gutman, B. Ruščič, N. Trinajstić, C. F. Wilcox, "Graph theory and molecular orbitals. XII. Acyclic polyenes", J. Chem. Phys., Vol. 62, pp. 3399-3405, 1975.

[7] I. Gutman, N. Trinajstić, "Graph theory and molecular orbitals. Total $\pi$-electron energy of alternant hydrocarbons", Chem. Phys. Lett., Vol. 17, pp. 535-538, 1972.

[8] I. Gutman, "Degree-based topological indices", Croat. Chem. Acta, Vol. 86, pp. 351-361, 2013.

[9] I. Gutman, “The energy of a graph”, Ber. Math. Stat. Sekt. Forschungsz. Graz. Vol. 103, pp. 1-22, 1978. 
[10] J. Liu, X. Li, "Hermitian-adjacency matrices and Hermitian energies of mixed graphs", Lin. Alg. Appl., Vol. 446, pp. 182207, 2015.

[11] Y. Lu, et al., "Hermitian-Randić matrix and Hermitian-Randić energy of mixed graphs", Journal of Inequalities and Applications, Vol. 54, 2017.

[12] N. J. Rad, et al., "Zagreb Energy and Zagreb Estrada Index of Graphs", MATCH Commun. Math. Comput. Chem. Vol. 79, pp. 371-386, 2018.

[13] J. A. Rodríguez, "A spectral approach to the Randić index”, Lin. Alg. Appl., Vol. 400, pp. 339-344, 2005.

[14] J. M. Rodríguez, J. M. Sigarreta, "Spectral properties of geometric-arithmetic index", Appl. Math. Comp., Vol. 277, pp. 142-153, 2016.

[15] J. M. Rodríguez, J. M. Sigarreta, "Spectral study of the Geometric-Arithmetic Index”, MATCH Commun. Math. Comput. Chem., Vol. 74, pp. 121-135, 2015.

\section{Author's Profile}

Dr Ankur Bharali is a faculty of Department of Mathematics, Dibrugarh University for last 9 years. His broad areas of interest include Graph Theory and Complex Network. He completed his master degree in Maths \& Computing from IIT Guwahati and obtained his $\mathrm{PhD}$ from Dibrugarh University. He authored a book and many research articles in reputed

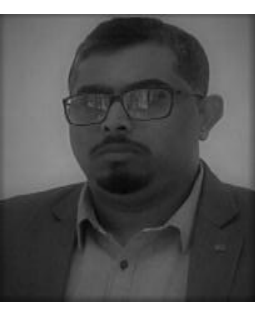
international journals. 